\title{
INCLUSÃO SOCIAL E O ALUNO COM SÍNDROME DE DOWN: UM ESTUDO DE CASO NAS AULAS DE EDUCAÇÃO FÍSICA
}

\author{
Maria Luiza Tanure Alves \\ Universidade Estadual de Campinas, Campinas, São Paulo, Brasil \\ Edison Duarte \\ Universidade Estadual de Campinas, Campinas, São Paulo, Brasil
}

\begin{abstract}
Resumo
O estudo tem como objetivo analisar a inclusão social de crianças com deficiência intelectual nas aulas de educação física escolar. Foi aplicado o teste sociométrico no início e final do ano letivo para identificação e análise do status social do aluno com deficiência intelectual. Participaram do estudo um aluno com síndrome de Down da $3^{\text {a }}$. série do ensino fundamental e seus 32 colegas de classe. $\mathrm{O}$ aluno com SD encontrou dificuldades para interação com seus pares durante as aulas de educação física. O aluno com SD demonstrou limitações importantes na estruturação de relações sociais, com relações restritas em quantidade.
\end{abstract}

Palavras-chave: Síndrome de Down. Educação Física e Treinamento. Educação Especial.

\section{Introdução}

inclusão de alunos com deficiência na escola tem como objetivo
principal a aprendizagem dos conteúdos propostos, bem como a inserção social do aluno em seu meio. A inclusão escolar não diz respeito somente à oportunidade em desempenhar as atividades propostas pelo professor em sala, mas também em participar e estruturar uma rede de relações sociais com seus colegas de classe (ODOM; DIAMOND, 1998; PLACE; HODGE, 2001; PIVIK; MCCOMAS; LAFLAME, 2002). Estes propósitos são de responsabilidade da equipe escolar como um todo, não cabendo apenas ao seu professor de sala de aula (STAINBACK; STAINBACK, 1999).

Para concretização da inclusão escolar, é necessário que as escolas e seus profissionais forneçam adaptações nos métodos de ensino, conteúdos, objetivos, materiais de aula, bem como na estrutura física para que esse aluno consiga atingir os objetivos de aprendizagem pre- 
viamente estabelecidos. Estes requisitos são amplamente defendidos pela legislação educacional brasileira em seus diversos documentos oficiais, tais como a Declaração de Salamanca de 1994; a Lei de Diretrizes e Bases da Educação Nacional de 1996; o Decreto Lei No. 3298 de Dezembro de 1999; as Diretrizes Nacionais para a Educação Especial na Educação Básica de 2001; o Plano Nacional de Educação - PNE de 2001; o Decreto $n^{\circ} 186$ de julho de 2008; e finalmente a Política Nacional de Educação Especial na Perspectiva da Educação Inclusiva de 2008 (ALVES; DUARTE, 2011).

Pesquisas no âmbito da interação social do aluno com deficiência descrevem que esta se desenvolve principalmente em um ambiente inclusivo (ODOM; DIAMOND, 1998; PLACE; HODGE, 2001). No entanto, Butler e Hodge (2004) relatam que são reduzidas as pesquisas que demonstram que a interação entre crianças com e sem deficiência nas aulas de educação física seja positiva e capaz de proporcionar sentimentos de aceitação e amizade para as mesmas. Em seu estudo sobre as relações sociais estruturadas pela criança com deficiência nas aulas de educação física escolar, Place e Hodge (2001) descrevem que as interações entre essas e seus colegas sem deficiência acontecem de forma reduzida. Os autores buscaram analisar a inclusão social de alunos com deficiência nas aulas de educação física através da aplicação de entrevistas semi-estruturadas e análise de vídeo das aulas observadas. Foi observado que os alunos com deficiência apresentaram relações sociais limitadas com seus pares sem deficiência, devido principalmente a distância espacial existente entre as partes, visto que o grupo de alunos com deficiência encontrava dificuldades para chegar ao local de aula.

Nesse sentido, Batista e Enumo (2004), em estudo realizado sobre as interações sociais de crianças com deficiência intelectual, descrevem a ocorrência de relações sociais limitadas entre estes e seus colegas de classe. Os alunos com deficiência intelectual participantes do estudo não foram totalmente aceitos por seus pares, indicando baixa freqüência de aceitação e maior nível de rejeição pelos colegas. Os mesmos foram incluídos fisicamente no ambiente, porém sem estabelecimento de vínculos sociais. Ellis, Wright e Cronis (1996) descrevem resultados semelhantes a estes em estudo realizado com crianças com deficiência intelectual para caracterizar as relações instituídas nas aulas de educação física. Como resultado os autores descrevem a re- 
duzida interação desses alunos com seus pares no contexto-alvo do estudo.

No entanto, Butler e Hodge (2004) descrevem a ocorrência de interações positivas entre alunos com e sem deficiência nas aulas de educação física. Os autores realizaram estudo com objetivo de investigar as relações sociais construídas entre alunos com e sem deficiência nas aulas de educação física. Apesar de apresentarem caráter positivo com demonstrações de respeito, amizade e cooperação, as interações entre os alunos com e sem deficiência eram limitadas e com baixa freqüência.

Interações reduzidas entre alunos com e sem deficiência também foram encontradas por Goodwin e Watkinson (2000) em trabalho realizado sobre a experiência de alunos com deficiência nas aulas de educação física. Os alunos foram entrevistados sobre suas experiências e inclusão durante as aulas, demonstrando isolamento social associado a experiências de rejeição, negligência ou autopercepção como um objeto de curiosidade pelos demais. Para o aluno com deficiência, as experiências positivas nas aulas de educação física ocorrem quando há um senso de pertencimento ao grupo, participação total e possibilidade de benefício com o programa de atividades propostas (GOODWIN; WATKINSON, 2000).

Dessa forma, este estudo tem como objetivo principal analisar a participação social do aluno com deficiência intelectual, mais especificamente o aluno com Síndrome de Down (SD), durante as aulas de educação física escolar. É objetivo do estudo analisar a inclusão do aluno com SD através de suas relações sociais construídas. Este tipo de estudo possibilita uma análise do viés social da inclusão do aluno com SD dentro deste contexto específico das aulas de educação física, possibilitando aos professores da disciplina a melhor compreensão do tema e planejamento futuro de metas para a sua concretização.

\section{Método}

O estudo foi realizado através de um estudo de caso qualitativo. A coleta de dados foi precedida por um estudo piloto com finalidade de ajustar e avaliar os instrumentos de medida. $\mathrm{O}$ estudo foi submetido e aprovado pelo Comitê de Ética da Universidade Estadual de Campinas - UNICAMP com o protocolo número 140/2007. A pesquisa foi realizada em uma escola pública estadual situada no município de Campi- 
nas - SP. A referida unidade escolar é composta por turmas de $1^{\circ}$. a $4^{\circ}$. séries do ensino fundamental. As aulas de educação física escolar ocorreram com freqüência de duas vezes por semana e duração de cinquenta minutos.

\section{Participantes}

Para a análise concreta da inclusão de crianças com SD, foi necessária a utilização de todos os alunos de uma mesma classe como sujeitos da pesquisa. Como critério de inclusão no estudo, a classe deveria ter pelo menos um aluno com deficiência intelectual em seu quadro, e todos deveriam ter uma freqüência mínima de $75 \%$ nas aulas de educação física. Para participação no estudo, os alunos deveriam apresentar autorização de seus pais ou responsáveis através da assinatura do termo de consentimento e livre esclarecimento.

O estudo contou com a participação de sujeitos de uma classe do ensino fundamental, $3^{\circ}$. série, a qual apresentava um de seus alunos com SD. Este aluno possuía diagnóstico médico devidamente qualificado. Ao todo participaram da pesquisa 33 alunos (20 meninos e 13 meninas; 9,2 $\pm 0,61$ anos). $O$ aluno com SD é do sexo masculino, com 9 anos de idade. O mesmo não apresentava problemas para locomoção independente ou problemas de fala e linguagem.

Coleta de dados

Para a avaliação e análise da inclusão do aluno com SD foi empregado o teste sociométrico descrito e validado por Moreno (1994). Este consiste em um método de investigação com o objetivo de compreender as redes de vínculos sociais estruturados em grupos. Desta forma este instrumento é capaz de analisar as relações sociais em função das escolhas e rejeições manifestadas no grupo, fornecendo o status social individual, suas relações de afinidade e conflito, mutualidades e ausência de relações (MORENO, 1994).

O teste sociométrico deve ser aplicado através de questões onde o participante é impelido a escolher dentro do seu grupo aqueles aos quais gostaria de ter como companheiro segundo o critério escolhido para investigação. $\mathrm{O}$ critério de escolha consiste no objetivo para a escolha, podendo ser operativos, quando relacionados ao cumprimento de uma tarefa; ou afetivos, quando a parte emocional é enfatizada. É 
importante que os indivíduos se sintam impelidos a responder as questões de forma espontânea. Os critérios escolhidos devem incidir sobre fatores essenciais do grupo com uma significação clara para todos os seus membros (ALVES; DUARTE, 2010). Como resultado do teste sociométrico, os participantes são classificados dentro de cinco posições de status social: popular, rejeitado, ignorado, mediano e controverso. O status social traduz a posição social do indivíduo dentro do seu grupo, revelando seu nível de interação social com seus pares.

$\mathrm{O}$ teste sociométrico se traduz em um indicador de aceitação do sujeito pelo grupo através da sua escolha, rejeição ou indiferença pelos demais. A aplicação do teste sociométrico (MORENO, 1994) no referido estudo teve como objetivo analisar o status social da criança com SD dentro do seu grupo de sala de aula no contexto das aulas de educação física. Desta forma, o teste se traduz em um indicador de aceitação do sujeito pelo grupo através da sua escolha, rejeição ou indiferença pelos demais. O teste sociométrico teve também como meta analisar a dinâmica das relações construídas pelo aluno com SD durante o ano letivo.

Para tanto, o teste sociométrico consistiu em questionamentos capazes de expressar o nível de aceitação e rejeição aos colegas de classe pelos participantes do estudo. Como o contexto do estudo refere-se às aulas de educação física para crianças da $3^{\text {a }}$. série, foram realizadas duas questões fundamentais para todos os alunos da classe: 1) Com quem você mais gosta de brincar nas aulas de educação física?; 2) Com quem você não gosta de brincar nas aulas de educação física? Esses dois questionamentos tinham como meta a manifestação de escolhas e rejeições dentro do grupo. Utilizou-se o termo "brincar"1 ao invés de "realizar as atividades da aula" para melhor compreensão dos participantes, visto que estes ainda compreendem a aula de educação física como um momento de brincadeiras, mesmo que direcionadas pelo professor. Todos os sujeitos foram questionados individualmente e isoladamente em horário pré-determinado de acordo com a disponibilidade dos participantes.

O teste foi aplicado no começo e final do período letivo (Teste I e Teste II) para acompanhar as mudanças nas relações instituídas pelo

1-Ambas as terminologias foram testadas durante o teste piloto, prevalecendo o termo "brincar" devido à melhor compreensão e associação com o contexto das aulas de educação física pelos participantes do estudo. 
aluno com SD durante o ano. A quantidade de escolhas e rejeições realizadas pelos sujeitos não foram limitadas para que se pudesse respeitar a condição de espontaneidade defendida por Moreno (1994). Cada sujeito recebeu uma identificação numérica para manter o sigilo de sua identidade. A análise dos índices sociométricos foi realizada de acordo com o modelo bi-dimensional de Coie e Dodge (1983): 1) as escolhas e rejeições recebidas por cada sujeito foram tabuladas e padronizadas; 2) foram calculados os índices de impacto social (IS) e preferência social (PS); 3) os índices de IS e PS são padronizados; 4) os sujeitos são classificados de acordo com os índices obtidos. O impacto social consiste na visibilidade que o sujeito tem em seu grupo, e é calculado pela soma das escolhas e rejeições recebidas. O índice de preferência social diz respeito ao grau de empatia que a criança obtém junto aos seus colegas de classe (NEWCOMB; BUKOWSKI, 1983). Este é calculado através da diferença entre as escolhas e rejeições recebidas por cada sujeito. A classificação de cada sujeito dentro dos cinco tipos de status social é realizada de acordo com os seguintes critérios (MAASSEN; VERSCHUEREN, 2005): 1) Popular; grupo formado por todas os sujeitos com índice de preferência social maior que 1 , escolhas recebidas padronizadas maior que 0 , e rejeições recebidas menor 0 ; 2) Rejeitado; sujeitos com preferência social menor que -1, rejeições recebidas maior que 0 , e escolhas recebidas padronizadas menor que 0 ; 3) Ignorado; sujeitos com impacto social menor que -1, escolhas menor que zero, e rejeições menor que zero; 4) Controverso; sujeitos com impacto social maior que 1 , e com escolhas e rejeições recebidas maiores que 1;5) Mediano; sujeitos que não se encaixaram nos perfis anteriores.

Os dados da classificação sociométrica foram analisados pelo teste de Kolmogorov-Smirnov para verificar aderência a distribuição normal. Posteriormente foram utilizados o teste qui-quadrado, teste de Wilcoxon e teste de Mann-Whitney. O teste qui-quadrado foi utilizado para observar diferenças significantes entre os cinco grupos da classificação sociométrica no teste sociométrico I e teste sociométrico II. O teste de Wilcoxon e teste de Mann-Whitney analisaram respectivamente as alterações significantes na distribuição dos sujeitos em cada grupo entre o teste sociométrico I e teste sociométrico II, e diferenças na classificação sociométrica de meninos e meninas em cada teste. $\mathrm{O}$ teste de Wilcoxon ainda foi empregado para avaliar alterações na distribuição das meninas e meninos entre o teste sociométrico I e teste 
sociométrico II. O nível de significância adotado foi de $\mathrm{p} \leq 0,05$. Foi utilizado o software Bioestat para realização dos serviços computacionais.

\section{Resultados}

A ausência de limites na quantidade de escolhas e rejeições pelos sujeitos resultou em $2,27( \pm 1,15)$ escolhas no teste sociométrico I e $2,73( \pm 1,14)$ no teste sociométrico II. Quanto às rejeições, os sujeitos realizaram $1,46( \pm 1,12)$ no teste sociométrico I e $1,83( \pm 1,07)$ no teste sociométrico II. Os resultados obtidos com a classificação sociométrica estão representados na Tabela I. O teste de Kolmogorov-Smirnov indicou que os dados no teste sociométrico I $(\mathrm{p}<0,05)$ e teste sociométrico II $(p<0,01)$ não são aderentes à distribuição normal.

$\mathrm{O}$ teste qui-quadrado resultou em diferenças significantes entre os grupos em ambos os testes, $\mathrm{X} 2=24,35, \mathrm{p}<0,001$ (teste sociométrico I) e X2 $=44,98, p<0,0001$ (teste sociométrico II). No primeiro teste sociométrico os sujeitos foram julgados predominantemente como medianos $(53,3 \%)$ ou ignorados $(20 \%)$. Essa tendência se altera no segundo teste sociométrico com diminuição dos sujeitos considerados como ignorados $(15,6 \%)$ e aumento principalmente do número de medianos (59,3\%). Apesar da queda no número de sujeitos classificados como ignorados, este se ainda se mantém como o segundo maior grupo de sujeitos no teste sociométrico II.

Tabela 1: Classificação sociométrica de alunos do ensino fundamental por sexo

\begin{tabular}{|c|c|c|c|c|c|c|c|c|c|c|}
\hline & \multicolumn{4}{|c|}{ TESTE I* } & \multicolumn{6}{|c|}{ TESTE II** } \\
\hline & \multicolumn{2}{|c|}{ Meninos } & \multicolumn{2}{|c|}{ Meninas } & \multirow{2}{*}{$\begin{array}{c}\text { TOTAL } \\
\% \\
\end{array}$} & \multicolumn{2}{|c|}{ Meninos } & \multicolumn{2}{|c|}{ Meninas } & \multirow{2}{*}{$\begin{array}{c}\text { TOTAL } \\
\% \\
\end{array}$} \\
\hline & $n$ & $\%$ & $n$ & $\%$ & & $n$ & $\%$ & $n$ & $\%$ & \\
\hline POPULAR & 3 & 17,6 & 0 & 0 & 10 & 2 & 10 & 2 & 16,6 & 12,5 \\
\hline REJEITADO & 2 & 11,7 & 1 & 7,6 & 10 & 1 & 3.13 & 2 & 16,6 & 9,3 \\
\hline IGNORADO & 4 & 23,5 & 2 & 15,3 & 20 & 2 & 10 & 3 & 25 & 15,6 \\
\hline CONTROVERSO & 1 & 5,8 & 1 & 7,6 & 6,6 & 2 & 10 & 2 & 16,6 & 12,5 \\
\hline MEDIANO & 7 & 41,1 & 9 & 69,2 & 53,3 & 13 & 65 & 3 & 25 & 59,3 \\
\hline TOTAL & 17 & 100 & 13 & 100.0 & 100 & 20 & 100 & 12 & 100 & 100 \\
\hline
\end{tabular}

Resultados do testes de Wilcoxon indicaram não haver diferenças significantes entre a distribuição dos sujeitos em cada grupo no teste sociométrico I e teste sociométrico II $(\mathrm{p}=0,62)$. Em ambos os testes sociométricos não foram encontradas diferenças significantes na dis- 
tribuição dos sujeitos por sexo (teste de Mann-Whitney) em cada um dos status sociométricos (teste I $\mathrm{p}=0,21$; teste II $\mathrm{p}=0,93$ ), indicando que a classificação sociométrica não foi influenciada pelo sexo dos sujeitos. O teste de Wilcoxon também não indicou alterações significantes entre a classificação sociométrica dos meninos no teste sociométrico I e teste sociométrico II $(\mathrm{p}=0,15)$. O mesmo resultado foi encontrado na classificação sociométrica das meninas nos testes sociométricos I e II $(\mathrm{p}=0,76)$.

No teste I o aluno com SD foi classificado como ignorado com índices de PS $=-0,41$ e IS $=-1,3$. No teste II, o mesmo manteve o status de ignorado, porém apresentou alterações em PS $=0,41$ e IS = -1,27. $\mathrm{O}$ mesmo realizou duas escolhas e uma rejeição restringindo-se apenas a sujeitos do mesmo sexo. $\mathrm{O}$ aluno não foi escolhido por qualquer membro do grupo, porém foi rejeitado por um sujeito do mesmo sexo. O sujeito não apresentou reciprocidades em escolha ou em rejeição, bem como incongruências com o grupo. Dessa forma, o aluno não se envolveu em relações de díade, triângulos ou círculos sociais com sua turma.

No teste sociométrico II referente a este grupo (Figura II), o aluno com SD mantém seu status de ignorado com baixo IS (-1,27), apesar de sua PS $(0,41)$ apresentar aumento em relação ao teste I. O aluno realizou duas escolhas, restritas ainda a companheiros do mesmo sexo, e uma rejeição referente a um membro do grupo do sexo oposto. As escolhas e rejeições estruturadas neste segundo teste sociométrico não foram as mesmas apresentadas no primeiro teste. O mesmo foi escolhido por um colega de classe, o qual estabeleceu uma relação de reciprocidade. Não foram feitas rejeições a criança com SD pelos colegas de classe, não havendo assim reciprocidades em rejeição. Também não foram estabelecidas incongruências entre escolha e rejeição entre o mesmo e seus pares. Os resultados dos testes sociométricos indicam que o aluno apresentava no início do período letivo baixo grau empatia e visibilidade frente aos companheiros de classe. No segundo semestre houve um aumento no seu grau de empatia, porém este aumento não foi suficiente para alterar sua visibilidade no grupo e modificar seu status social frente aos colegas. 


\section{Discussão}

Os resultados encontrados demonstram falhas importantes na participação social dos alunos nas aulas de educação física, visto o número expressivo de alunos classificados como ignorados em ambos os testes sociométricos ( $20 \%$ no teste I e 15,6 no teste II). Pessoas classificadas como ignoradas são aquelas que, em comparação com os medianos, demonstram baixa agressividade e sociabilidade, pouca interação social, bem como poucas ações e características sociais positivas. Estes podem apresentar também altos níveis de timidez (COIE; DODGE, 1983; NEWCOMB; BUKOWSKI; PATEE,1993).

O aluno com SD também demonstrou limitações importantes na estruturação de relações sociais. Suas relações com os colegas de classe eram restritas em quantidade e marcadas pelos baixos índices de visibilidade social (IS) apresentados em ambos os testes sociométricos aplicados. Os métodos empregados não permitem mensurar se tal fato se deve as limitações impostas pela SD, aos conteúdos ministrados, metodologia de ensino utilizada nas aulas, bem como atitudes e comportamentos negativos seus ou de seus pares. A ocorrência de interações sociais limitadas também foi relatada nos estudos de Goodwin e Watkinson (2000), Place e Hodge (2001), Batista e Enumo (2004) e Butler e Hodge (2004). No entanto, Odom e Diamond (1998) descrevem que as interações da criança com deficiência com os colegas de classe ocorrem mais freqüentemente em ambientes inclusivos quando comparados com ambientes especializados.

Apesar da construção de relações com os colegas de forma limitada, o aluno com SD apresentou alterações nas relações estruturadas durante o ano letivo. As mudanças realizadas nas escolhas e rejeições entre o teste sociométrico I e II, bem como a estruturação de uma relação de reciprocidade no teste sociométrico II demonstra que o mesmo conseguiu desenvolver relações com colegas diferentes entre os períodos de aplicação do teste sociométrico. Porém estas alterações não podem ser entendidas como um aumento na rede de relações, pois estas não foram capazes de alterar positivamente o seu status social.

Os problemas para inclusão dos alunos na rede social da turma não dizem respeito somente aos alunos com deficiência. Os resultados encontrados demonstram que os conteúdos e atividades das aulas de educação física não foram capazes de incluir na rede de relações da turma outros alunos sem deficiência, visto que $30 \%$ dos alunos no tes- 
te I e $26,6 \%$ no teste II foram classificados como ignorados ou rejeitados. Isso demonstra que as dificuldades para inclusão social no âmbito escolar ultrapassam as barreiras da deficiência, e atingem alunos de toda a turma não classificados como tendo alguma necessidade educacional especial. Desta forma, os dados encontrados demonstram que as barreiras encontradas para a inclusão dos alunos nas aulas de educação física ultrapassam as limitações e necessidades especiais descritas e definidas nos documentos oficiais que regem a legislação educacional brasileira.

A inclusão do aluno com SD durante as aulas de educação física esbarra em dificuldades severas para promover a sua participação social junto à turma. Os resultados aqui descritos demonstram um prejuízo por parte do mesmo em estruturar relações sociais com seus pares durante as aulas de educação física. Este manteve durante todo o ano o status de ignorado, indicando sua baixa visibilidade e empatia junto aos seus colegas de classe. Apesar do seu status de ignorado, o aluno conseguiu estruturar uma relação de reciprocidade durante o ano letivo, bem como relações de troca com um reduzido número de colegas. No entanto os métodos aqui empregados não possibilitam analisar as causas para as limitações encontradas para estruturação de relações sociais durante as aulas de educação física. Essa limitação pode estar relacionada com falhas no processo pedagógico, limitações impostas pela própria $\mathrm{SD}$, bem como atitudes e comportamentos aversivos de seus colegas de classe.

No entanto, estes resultados não devem ser generalizados, pois o estudo apresenta algumas limitações como o 1) reduzido número de sujeitos com deficiência; 2) limitação apenas à uma unidade escolar do ensino fundamental; 3 ) não investigação das causas para a reduzida interação social. Para a avaliação da inclusão teria sido de grande valia a realização de entrevistas com os próprios alunos com deficiência intelectual para compreensão dos pontos positivos e negativos vivenciados por este.

Ainda são poucos os estudos que procuram investigar a concretização da inclusão de alunos com deficiência na rede regular de ensino a partir da análise da construção de relações sociais. Futuros estudos devem analisar as causas das limitadas relações sociais estruturadas pela criança com deficiência intelectual durante as aulas de educação física, avaliar a autopercepção sociométrica desses alunos, bem como analisar a estabilidade da classificação sociométrica durante os vários 
anos escolares. Estudos semelhantes a este devem ser realizados com um número maior de alunos com deficiência como sujeitos, analisando a inclusão em diversas escolas e em diferentes regiões brasileiras. É de grande valia também a avaliação das causas para a limitação na construção de relações sociais limitadas. Dessa forma, poderão ser observados e elucidados as possíveis falhas presentes no sistema educacional brasileiro para a inclusão de alunos com deficiência intelectual. Tal elucidação é de fundamental importância para alteração e elaboração de novas políticas públicas para melhoria do processo inclusivo.

Para melhor elucidação das dificuldades encontradas durante a inclusão de alunos com deficiência é importante que sejam ouvidos os próprios alunos e seus pais (GOODWIN; WATKINSON, 2000; COOK; SWAIN; FRENCH, 2001; PIVIK; MCCOMAS; LAFLAME, 2002; HUTZLER et al., 2002). Outro ponto importante para a exclusão é falta de conhecimento do professor para trabalhar com alunos com deficiência (ROLL-PETTERSON, 2001; HUTZLER et al., 2002; PIVIK; MCCOMAS; LAFLAME, 2002; BLOCK; OBRUSNIKOVA, 2007). Essa falta de conhecimento geralmente faz com o que o professor tenha uma visão negativa sobre o processo e acabe criando experiências negativas para o seu aluno (BLOCK; OBRUSNIKOVA, 2007). A inclusão pode ter sucesso e não causar prejuízos para seus alunos desde que os alunos com deficiência recebam o devido suporte material e profissional especializado (BLOCK; ZEMAN, 1996; PLACE; HODGE, 2001; BLOCK; OBRUSNIKOVA, 2007).

\title{
SOCIAL INCLUSION AND THE STUDENT WITH DOWN SYNDROME: A
} CASE STUDY IN PHYSICAL EDUCATION CLASSES

\begin{abstract}
The aim of this study was to investigate the social inclusion of children with intelectual disability in physical education classes. At the beginning and at the end of the year sociometric test was applied to analyze the social status of a student with intellectual disability. Thirty-three third grade students, one with Down Syndrome (DS) student and 32 peers, served as subjects for this study. The SD student had difficulties to Interact with peers during physical education classes. Further, SD student presented important limitations in building social relationships, having built few of them.
\end{abstract}

Keywords: Down Syndrome. Physical Education and Training. Education, Special. 
LA INCLUSIÓN SOCIAL Y ALUMNOS CON SÍNDROME DE DOWN: UN ESTUDIO DE CASO EN LAS CLASES DE EDUCACIÓN FÍSICA

\section{Resumen}

El estudio tiene como objetivo analizar la inclusión de los niños con discapacidad intelectual en las clases de educación física. Test sociométrico se aplicó al inicio y al final del año para la análisis de la inclusión. El estudio incluyó a un estudiante con síndrome de Down de la escuela primaria y sus compañeros de clase 32. El estudiante con síndrome de Down tenían dificultades para interactuar con sus compañeros durante las clases de educación física. El estudiante con síndrome de Down mostraron limitaciones significativas en la estructuración de las relaciones sociales, las relaciones con cantidad limitada.

Palabras clave: Síndrome de Down. Educación y Entrenamiento Físico. Educación Especial.

\section{Referências}

ALVES, M. L. T.; DUARTE, E. O Processo Inclusivo nas Aulas de Educação Física: Um Estudo sobre o Teste Sociométrico. Revista da Educação Física/UEM, Maringá, v.21, n. 3, p. 479-491, 2010.

- Os caminhos percorridos pelo processo inclusivo de alunos com deficiência na escola: uma reflexão dos direitos construídos historicamente. Revista Educação Especial, Santa Maria, v. 24, n. 40, p. 207-218, maio/ago., 2011.

BATISTA, M. W.; ENUMO, S. R. F.. Inclusão Escolar e Deficiência Mental: Análise da Interação Social entre Companheiros. Estudos de Psicologia, Natal, v. 9, n. 1, p.101-111, 2004.

BLOCK, M.; OBRUSNIKOVA, I. Inclusion in Physical Education: A Review of Literature from 1995-2005. Adapted Physical Activity Quarterly, Illinois, v. 24, 103-124, 2007.

BLOCK, M.; ZEMAN, R. Including students with disabilities in regular physical education: effects on nondisabled children. Adapted Physical Activity Quarterly, Illinois,v. 13, n.1, p.38-49, 1996.

BUTLER, R.; HODGE, S. Social Inclusion of Students with Disabilities in Middle School Physical Education Classes. Research in Middle Level Education Online, Ohio, v. 27, n. 1, p. 1-10, 2004. 
COIE, J. D.; DODGE, K. A. Continuities and Changes in Children's Social Status: A Five-Year Longitudinal Study. Merril-Palmer Quarterly, Detroit, v. 29, n.3, 261-282, 1983.

COOK, T.; SWAIN, J.; FRENCH, S. Voices from Segregated Schooling: Towards an Inclusive Education System. Disability and Society, Newcastle, v.16, n. 2, p. 293-310, 2001.

ELLIS, D.; WRIGHT, M.; CRONIS, T. A Description of the Instructional and Social Interactions of Students with Mental Retardation in Regular Physical Educations Settings. Education and Training in Mental Retardation and Developmental Disabilities, Arlington, v. 31, p. 235-241, set., 1996.

GOODWIN, D.; WATKINSON, J. Inclusive Physical Education from the Perspective of Students with Physical Disabilities. Adapted Physical Activity Quarterly, Illinois, v. 17, 144-163, 2000.

HUTZLER, Y. et al. Perspectives of Children with Disabilities on Inclusion and Empowerment: Supporting and Limiting factors. Adapted Physical Activity Quarterly, Illinois, v. 19, 300-317, 2002.

MAASSEN, G.; VERSCHUEREN, K. A Two-Dimensional RatingsBased Procedure for Sociometric Status Determination as an Alternative to the Asher and Dodge System. Merril-Palmer Quarterly, Detroit, v.51, n. 2, p. 192-212, Abril, 2005.

MORENO, J. L. Quem Sobreviverá: Fundamentos da Sociometria, Psicoterapia de Grupo e Sociodrama. Dimensão Editora: Goiânia, 1994.

NEWCOMB, A.; BUKOWSKI, W. Social Impact and Social Preference as Determinants of Children's Peer Group Status. Developmental Psychology, Washington, v. 19, n.6, p. 856-867, 1983.

NEWCOMB, A., BUKOWSKI, W.; PATTEE, L. Children's peer relations. A meta-analytic review of popular, rejected, neglected, controversial, and average sociometric status. Psychological Bulletin, Washington, v.113, n.1, p. 99-128, 1993. 
ODOM, S.; DIAMOND, K.; Inclusion of Young Children with Special Needs in Early Childhood Education: The Research Base. Early Childhood Research Quarterly, Fairfax, v. 13, n.1, p.3-25, 1998.

PIVIK, J.; MCCOMAS, J.; LAFLAME, M.. Barriers and Facilitators to Inclusive Education. Exceptional Children, Arlington, v. 69, n. 1, p.97-107, 2002.

PLACE, K.; HODGE, S. Social Inclusion of Students with Psysical Disabilities in General Physical Education: A Behavioral Analyses. Adapted Physical Activity Quarterly, Illinois, v. 18, p.389-404, 2001.

ROLL-PETTERSON, L. Teacher Perceptions of Supports and Resources Deficiênciaded in Regard to Pupils with Special education Deficiênciads in Sweden. Education and Training in Mental Retardation and Developmental Disabilities, Arlington, v. 36, no.1, p.42-54, 2001.

STAINBACK, W.; STAINBACK, S. Colaboração, Rede de Apoio e Construção de Comunidade. In: __ Inclusão: Um Guia para Educadores. Porto Alegre: Artes Médicas, 1999. p. 223-232.

Recebido em: 19/12/2012

Revisado em: 05/03/2013

Aprovado em: 12/04/2013

\section{Endereço para correspondência}

edison@fef.unicamp.br

Edison Duarte

Universidade Estadual de Campinas

FACULDADE DE EDUCACAO FISICA

DEPARTAMENTO DE ESTUDOS DA ATIVIDADE FÍSICA ADAPTADA.

Cidade Universitária

Barão Geraldo

13083-851 - Campinas, SP - Brasil - Caixa-postal: 6134 